\title{
DIFERENCIACIÓN DIALECTAL POR MEDIO DE PRONOMBRES: UNA COMPARACIÓN DEL USO DE TÚ Y USTED EN ESPAÑA Y MÉXICO
}

\section{INTRODUCCIÓN}

El hecho de que existan diferencias de tratamiento entre los habitantes de dos países distintos no sorprende al que ha viajado internacionalmente. Desde los gestos corporales hasta la manera de entrar en una tienda, es preciso conocer las normas del lugar en donde se encuentre. El sociolingüista, además, se interesa en estas diferencias culturales y el efecto que acompaña su aparición en la lengua hablada y en otros aspectos conversacionales. Gumperz resume muy hábilmente la amplia influencia que ejercen estas divergencias:

Socio-cultural conventions affect all levels of speech production and interpretation from the abstract cultural logic that underlies all interpretation to the division of speech into episodes; from their categorization in terms of semantically relevant activities and interpretive frames, to the mapping of prosodic contours into syntactic strings and to selection among lexical and grammatical options ${ }^{1}$.

En este sentido, España y México no son excepciones y las diferencias existentes entre ellos son bastante fáciles de percibir. Como ilustración de unas divergencias representativas, aunque debido a las limitaciones del papel sean fundamentalmente de tipo lingüístico, obsérvense las siguientes situaciones, presenciadas por el autor en los dos países:

Escena: una panadería en Alicante, España. Entra una mujer de 30 años quien es atendida por una dependienta de 20 .

${ }^{1}$ GuMperz 1982, p. 186. 
Cliente: Hola.

Dependienta: Hola, ¿qué quieres?

Cliente: Ponme dos barras de pan y un paquete de galletas de chocolate.

Dependienta: (se lo entrega) Aquí tienes, ¿algo más?

Cliente: No nada, dime cuánto es.

Dependienta: Son 150 pesetas.

Cliente: (le da el dinero) Aquí tienes.

Dependienta: Vale, gracias.

Clienta: Hasta luego.

Dependienta: Adiós.

Escena: otra panadería en México, D. F. Entra una mujer de 30 años quien es atendida por una dependienta de 20 .

Dependienta: Buenas tardes.

Cliente: Buenas tardes.

Dependienta: ¿En qué le puedo servir?

Cliente: ¿Podría darme Ud. una docena de tortas, por favor?

Dependienta: Cómo no, señorita (se las entrega), aquí tiene, ¿quisiera otra cosa?

Cliente: No, gracias, esto será todo.

Dependienta: Muy bien, son 3500 pesos.

Cliente: (le da el dinero) Aquí tiene Ud.

Dependienta: Gracias, que tenga Ud. buenas tardes.

Cliente: Igualmente, hasta luego.

De estos dos pequeños diálogos puede extraerse mucha información acerca de la naturaleza de las diferentes formas de tratamiento en los dos países. Primero, el saludo de los interlocutores es, en el caso español, un elemento léxico más informal que en la situación mexicảna. La petición que se hace de la mercancía también demuestra variación en los dos contextos, es un mandato directo para las españolas (igual que la petición de la cliente para el costo) y una pregunta indirecta de parte de las mexicanas. Finalmente, y quizás lo más prominente tanto para los hablantes como para el sociolingüista, el trato pronominal es diferente: en tanto que las españolas se hablan de "tú", las mexicanas se tratan de "Ud.".

Es este último contraste, es decir, la variabilidad de los pronombres "tú" y "usted" (de aquí en adelante abreviados por las iniciales "T/V', T representando "tú" y V "usted") existente entre hablantes españoles y hablantes mexicanos, además de algunos datos sobre las diferencias verbales en los estilos español y mexicano de conversación ya expuestos en otra ocasión (Schwenter 
1991), lo que me ha impulsado a proponer la presente investigación. En ella, intentaré ofrecer una muestra de la sistematización que alcanzan estos dos pronombres al ser examinados en relación con los clásicos parámetros sociológicos de edad, sexo y clase social, los cuales también han gozado de empleo muy extendido en estudios de otras lenguas que comparten la distinción pronominal que ejemplifican "T/V" (cf. Bates \& Benigni 1975). Adicionalmente, el presente estudio demostrará, a un nivel más específico, las reglas y convenciones que acata el uso pronominal en las dos naciones.

Sin embargo, en esta etapa del trabajo, debería notarse que no pretende efectuarse ningún intento de proporcionar una imagen definitiva de todas las facetas del trato pronominal entre España y México. Al igual que toda investigación sociolingüística, los resultados que se obtienen sólo reflejan una pequeña muestra de la población entera, de la cual luego extrapolamos nuestras conclusiones a grupos más amplios (Labov 1972). Así, pues, este estudio únicamente recoge informantes de una sección de las sociedades española y mexicana, la del nivel socioeconómico medio, y de dos lugares particulares dentro de los dos países, Alicante y México, D. F. No obstante, el hecho de que comparemos dos grupos más o menos homólogos, que han sido seleccionados e investigados según las mismas técnicas, permite que los resultados acumulados en esta investigación sean válidos y confiables para nuestras metas, esto es, el contraste de las muestras estudiadas y la revelación de los factores que contribuyen a este contraste ${ }^{2}$.

\section{Trabajos antecedentes de $T / V$}

El famoso estudio de Brown y Gilman (1960) abrió paso para la investigación de los sistemas de tratamiento pronominal como fenómeno lingüístico, sociológico y sociolingüístico. Brown y Gilman examinaron varios idiomas indoeuropeos ya que tanto en aspectos sincrónicos como diacrónicos exhiben la distinción pronominal $\mathrm{T} / \mathrm{V}$, y descubrieron que la selección de uno u otro de estos pronombres no es un proceso ad hoc. Al contrario, la elec-

${ }^{2}$ Doy mis más sinceras gracias a Sylvia Trujillo de Serradell, Ana Aliaga Mulero, "La Yaya", y a todos los que participaron en la investigación. También, por supuesto, quiero agradecer a mis padres y, finalmente, a Araceli Schwenter, quien tiene diez veces más paciencia que yo. 
ción entre $\mathrm{T} / \mathrm{V}$ se encuentra, en todos los casos, bajo la regulación de un conjunto de principios que consideran la covariación del pronombre utilizado, de un lado, y, del otro, el estatus objetivo y relaciones solidarias que existen entre los interlocutores ${ }^{3}$.

Brown y Gilman, por medio de su análisis, postularon dos clases de construcciones hipotéticas nombradas "semánticas" por las cuales se gobierna la elección pronominal. La primera de éstas, la "semántica de poder", como lo implica su nombre, explica el tratamiento no recíproco entre personas desiguales en cuanto a la autoridad. El poder propuesto por los autores hace su aparición en situaciones entre dos interlocutores en las que uno es capaz de controlar la conducta del otro, ya sea esta capacidad a base de riqueza, fuerza física, clase social o edad ${ }^{4}$. La "semántica de solidaridad", contrariamente, es recíproca o simétrica, y tiene su fundamento en las similitudes que se encuentran entre los hablantes. El caso de solidaridad en que los dos participantes emplean $\mathrm{V}$, además, se origina como consecuencia de la igualdad de clasificación social o de edad que se comparte, en combinación con una carencia mutua de familiaridad. El T recíproco ocurre en contextos de equivalencia social o de edad y familiaridad anterior, haciéndose más fuerte dentro de relaciones simétricas entre hermanos, e hipotéticamente alcanza niveles máximos entre gemelos ${ }^{5}$. El descubrimiento general derivado de la investigación estableció la tendencia creciente, al menos en las lenguas analizadas por los autores, para la regulación de la "semántica de solidaridad" sobre la selección de T/V, al mismo tiempo que predijo la virtual abolición de selección no recíproca conducida por la "semántica de poder"' en el porvenir de estos sistemas de tratamiento pronominal ${ }^{6}$.

La investigación sobre el uso de T/V en España, muy escasa, se ha limitado completamente a estudios del tipo entrevista/cuestionario (cf. Labov 1984). También, estos trabajos han fijado su enfoque en un segmento muy restringido de la población, estu-

3 Wainerman 1976, p. 48.

${ }^{4}$ Brown \& Gilman 1972, p. 255.

${ }^{5}$ Ibid., p. 258.

${ }^{6}$ Brown y Gilman, en verdad, no predijeron un periodo fijo de tiempo en el cual la "semántica de poder" desaparecería. Sin embargo, ellos sí notaron el cambio rápido al empleo del $\mathrm{T}$ recíproco en las lenguas europeas que estudiaron. Los hallazgos de otros estudios sobre España (FoX 1969, BORREgo Nieto et al. 1978, Aguado Candanedo 1981) e Italia (Bates \& Benigni 1975) han corroborado esta tendencia hacia T. 
diantes de colegio y universidad, con edades comprendidas entre 10 y 25 años. Fox (1969) encontró que escolares de toda edad reemplazaban el empleo de pronombres asimétricos por tratamientos solidarios, ya sea esta nueva utilización entre familiares y amigos (T), o mayores y desconocidos (V). Los descubrimientos de Fox proporcionaron un fuerte apoyo para las hipótesis de Brown y Gilman (1960). Borrego Nieto et al. (1978) confirmaron la potente influencia tanto de clase social como de edad sobre la elección pronominal de universitarios en Salamanca, y sintetizaron sus conclusiones en forma de diagramas de informática parecidos a los que fueron introducidos al campo del trato pronominal por Ervin-Tripp (1969). El estudio de Alba de Diego y Sánchez Lobato se destaca por su refutación de las observaciones de Beinhauer, quien opinaba que "el tratamiento que se da a sujetos depende en cada caso del grado de intimidad, pero hay que tener en cuenta que a los padres, tíos, tías y parientes políticos se les trata muy a menudo en tercera persona" 7 . Los investigadores, sin embargo, presentaron datos muy convincentes en contra de las creencias de Beinhauer. Finalmente, Aguado Candanedo (1981) empleó la encuesta de Barrego Nieto et al. para un examen del uso de universitarios de Bilbao. Sin ser sorprendentes, los resultados de Aguado Candanedo fueron bastante parecidos a los de sus colegas de Salamanca ${ }^{8}$.

Ahora bien, con referencia a la investigación del sistema $T / V$ en México, que yo sepa, sólo existen dos trabajos que se han dedicado al fenómeno sociolingüístico bajo inspección. Lastra de Suárez investigó la relación entre el uso pronominal y los factores sociológicos de edad y clase social en la capital mexicana. Ella resume así los hallazgos del corto estudio:

... podemos decir que el tratamiento asimétrico va disminuyendo en la ciudad de México. El uso recíproco de tú va en aumento, sobre todo dentro de la familia, pero también fuera de ella, ya que se emplea inclusive para dirigirse a maestros y sacerdotes. Asimis-

7 Beinhauer 1978 , p. 30.

${ }^{8}$ Un resultado muy interesante del estudio de Aguado Candanedo es que los profesores de Bilbao reciben más trato de $\mathrm{T}$ que los profesores de Salamanca. Unos informantes españoles familiarizados con las universidades del país, a quienes enfrenté con este hecho, me aseguraron que se debe al mayor prestigio nacional que se otorga a la Universidad de Salamanca. 
mo el uso recíproco de tú está sustituyendo al de usted, como en el caso de las conversaciones entre compradores y vendedores ${ }^{9}$.

El único otro estudio que trata el empleo de T/V en el ambiente mexicano es el de Keller (1974). Sin embargo, esta investigación se limitó a la consideración del tratamiento existente dentro de relaciones de compadrazgo. Dado que tal relación social no se da en España, el presente estudio no la examinará, por lo que no haré ninguna otra mención del trabajo de Keller ${ }^{10}$.

El estudio del sistema hispanoparlante pronominal T/V desde un punto de vista comparativo, es decir, entre dos "comunidades de habla" (cf. Gumperz 1968), también se caracteriza por una escasez de investigación académica. El instruido trabajo de Solé (1970) inspeccionó las divergencias existentes en el uso de $\mathrm{T} / \mathrm{V}$ entre hablantes de tres países que ejemplificaban tres etapas distintas de desarrollo industrial: Puerto Rico (sociedad agrícola), Perú (sociedad en vías de transición) y Argentina (sociedad moderna). Por medio de la interacción entre el nivel social y la utilización generalizada de T/V, llegó Solé a conjeturar algunas correlaciones elementales entre estos factores. Básicamente, se reveló que los tres países conservaron su orden de industrialización al considerar los pronombres. Esto es, Puerto Rico mostró más "arcaísmo y tradicionalismo" que Perú, mientras que la Argentina, en cambio alcanzó un nivel de solidaridad de T/V que simbolizaba la sociedad más avanzada11. Por último, la investigación de Marín (1972) ${ }^{12}$ presentó una comparación de

${ }^{9}$ Lastra de Suárez 1972, p. 215.

${ }^{10}$ En España sí existe el elemento léxico "compadre" como forma de tratamiento entre hombres de edad mayor. Sin embargo, su uso no señala ninguna connotación social, sino que se emplea de una manera semejante a la de un apodo.

11 SOLÉ 1970, pp. 185-188.

${ }^{12}$ El trabajo de Marín no parece presentar datos confiables ni en la actualidad, ni quizá en la época en que se publicó. Como demostración, considérese la siguiente conclusión a que llega Marín (1972, p. 907): "Usted [tiene] cierta mayor tendencia a ser sustituido por tú en Hispanoamérica que en España. En este país es frecuente incluso el caso de personas que llevan muchos años de trato sin llegar por ello a apear el $V d$., como el de muchos señores que se reúnen a diario en la tertulia de café"'. Obviamente, como comprueban los datos del presente estudio, la primera oración del comentario de Marín queda definitivamente fuera de lugar. Además, según mis informantes españoles mayores, muy difícilmente se encuentra tal caso del uso de $\mathrm{V}$ entre gente que se conoce, aun hace 20 años. 
$\mathrm{T} / \mathrm{V}$ entre estudiantes latinoamericanos y españoles que asistían a la Universidad de Toronto. Descubrió Marín que no había mucha diferenciación entre los americanos y los europeos en cuanto al empleo de los pronombres, y que los sistemas de tratamiento, para todos los informantes, se encontraban dentro de un proceso de cambio en el que estaba surgiendo alguna incertidumbre sobre la forma apropiada ${ }^{13}$.

A partir de la base provista por los trabajos arriba revisados, la presente investigación se aprovecha de la falta tanto de estudios actuales de $\mathrm{T} / \mathrm{V}$ en español, como los de enfoque comparativo, mexicano o, incluso, español, en un intento de ofrecer algunos datos beneficiosos para la sociolingüística. Aunque de naturaleza experimental, este estudio seguirá el tipo de investigación de Brown y Gilman con la meta no sólo de revelar los componentes determinantes del empleo y elección pronominal, sino también la variación clave de reciprocidad existente entre los grupos español y mexicano bajo observación. Así, se espera que la exploración aquí emprendida del tema proporcione un fundamento que podrá aplicarse al estudio futuro del tópico dinámico de los sistemas pronominales $\mathrm{T} / \mathrm{V}$.

\section{Metodología}

Cuarenta informantes, veinte de cada nacionalidad y diez de cada sexo dentro de los dos grupos, fueron encuestados en su lugar de residencia. Todos los españoles son habitantes actuales de la ciudad de Alicante, una moderna y creciente urbe que se sitúa unas dos horas en coche al sur de Valencia ${ }^{14}$. Los mexicanos encuestados residen actualmente en la ciudad de México, el centro cultural y económico del país. Además, por medio de la aplicación de varios criterios corroborados por mexicanos y españoles no participantes en el estudio, todos los informantes fueron seleccionados de lo que determinamos ser la clase socioeconómica media de los dos países, clasificación que, sin duda, es muy parecida en las sociedades española y mexicana. Por último, fue

13 Ibid., 1972, pp. 906-907.

14 Bien es sabido que en Alicante algunas personas también hablan el valenciano, lengua para unos, y dialecto del catalán para otros. Cualquiera sea su naturaleza, en esta investigación se intentó no incluir a valencianohablantes en la muestra, en tanto que la influencia de otro sistema T/V puede ejercer influjo sobre el del castellano. 
requerido que cada informante tuviera entre 26 y 50 años de edad. Esta condición se empleó por cuatro razones: primera, a causa de la conveniencia y facilidad con las que se encontraron bastantes informantes; segunda, por el hecho ya aludido de que toda la investigación publicada sobre el uso de T/V en España ha tratado las edades menores; tercera, puesto que esta sección de la población es la más grande, tendría contacto con una variedad mayor de situaciones sociales; finalmente, el análisis de este grupo permitiría el examen del efecto tanto de la juventud como de la ancianidad sobre la elección pronominal, como veremos en el apartado 4.2.

El cuestionario utilizado constó de 65 preguntas $^{15}$, de las cuales cada una representa una "identidad posicional" (cf. Irvine 1979), a quien los informantes respondieron marcando $\mathrm{T} o$ $V$. Las respuestas de "a veces $T$ ", "a veces $V$ " o "no sé", utilizadas muy a menudo por los investigadores de $\mathrm{T} / \mathrm{V}$, no se pusieron a la disposición de los encuestados, en tanto que la situación verdadera de la vida real los forzaría a que contestaran, ya que en castellano no es posible ocultar el tratamiento a no ser que no se diga un verbo. Las preguntas fueron formadas en cuatro grupos, el primero pidiendo sólo el tratamiento dado a tal persona, el segundo solicitando tanto el tratamiento dado como el recibido de miembros de la familia, el tercero, el dado y recibido de ciertas personalidades y el cuarto demandando el dado cuando otra persona ha abierto la situación con $\mathrm{T} o \mathrm{~V}$. También, debe notarse que las identidades posicionales fueron presentadas con tres elementos de información sobre el interlocutor ficticio: el sexo de la persona, su edad y el puesto de trabajo que ocupa (por ejemplo, médico, dependienta) o el estatus social o familiar (por ejemplo, vecino, abuela). Por fin, la mayor parte de la gente "inventada" para el cuestionario incorporó personalidades que los encuestados desconocían, con las obvias excepciones de las personas incluidas en el apartado sobre la familia (véase el apartado 4.5).

${ }^{15}$ Reconozco que existen desventajas al utlizar únicamente cuestionarios para la recolección de datos. También estoy de acuerdo con las precisiones metodológicas de LABOV (1984), quien afirma que los cuestionarios/entrevistas y la observación deberían utilizarse juntos para extraer lo más posible de los datos con la menor probabilidad de errores. No obstante, en cuanto a los estudios de $\mathrm{T} / \mathrm{V}$, tal combinación de métodos resulta difícil en la práctica, puesto que información adecuada sobre las personas estudiadas, especialmente la de índole sociológica, no puede recogerse en cortos periodos de tiempo. 


\section{Resultados}

Porcentajes de respuestas $\mathrm{T} / \mathrm{V}$ fueron calculados para los factores sociológicos de sexo, edad y clase social, además del total T/V para todas las preguntas. Tanteos para uso pronominal con miembros de la familia también son reportados por medio de porcentajes, aunque no se han incluido datos para el empleo que reciben los informantes ya que muy pocas respuestas (menos de $1 \%$ ) fueron de tipo V. Sin embargo, una cuenta de reciprocidad (cf. infra) sí fue hecha para examinar la variación entre los pronombres "dados" y "recibidos".

Al final fueron analizadas las encuestas de 18 de los 20 informantes de cada país. Esta reducción se hizo necesaria al considerar dos cuestionarios españoles en que parecía que los participantes no habían entendido las instrucciones, y una encuesta mexicana rellenada por una persona que resultó no ajustarse a las limitaciones de la muestra. También, nótese que dentro de las tablas estadísticas que se presentan a continuación la abreviatura pronominal $\mathrm{T} / \mathrm{V}$ es revertida a la forma española extendida de "tú" y "usted"' o las mayúsculas "TÚ" y "UD.". Este cambio se ha efectuado con el propósito de aclarar la lectura de estos diagramas.

\subsection{Totales de $T / V$}

Como primera medida, se computó el porcentaje final del empleo pronominal de los informantes, utilizándose como metodología la adición de los totales de cada una de las cuatro secciones del cuestionario arriba mencionadas. Para cada uno de los dos grupos, tanto mexicano como español, el número total de preguntas era 1170 , esto es, 65 preguntas/encuesta por 18 informantes. Los resultados obtenidos se muestran en la tabla 1.

TABLA 1

Empleo de $T / V$ por nacionalidad, datos globales

\begin{tabular}{lcc}
\hline & $T U$ & $U D$. \\
\hline mexicanos & $32 \%(374)$ & $68 \%(796)$ \\
españoles & $56 \%(656)$ & $44 \%(518)$ \\
\hline
\end{tabular}


Arriba encontramos una diferencia bastante grande entre las dos nacionalidades en cuanto al uso cabal de los pronombres T/V. Este resultado contrasta fuertemente con el de Marín (1972), quien llegó a la conclusión de que no había mucha diferenciación del empleo de $\mathrm{T} / \mathrm{V}$ entre españoles y latinoamericanos. Si los resultados suyos tienen validez, en vista de los porcentajes de la tabla 1, debe ser que otros hispanoamericanos siguen reglas distintas de las mexicanas. Por este sencillo razonamiento, una metodología que intenta sintetizar los sistemas de hablantes de diversos países en comparación con un sistema propio de unos hablantes de un solo país no resulta ser suficiente, puesto que no presenta un retrato verídico de ningún dialecto en particular. Y como los investigadores del idioma español sabemos, Latinoamérica no comprende una área dialectal, sino muchas. Además, es muy probable que amplios cambios del empleo de T/V en España se hayan producido desde hace 19 años cuando Marín hizo su estudio. Sin embargo, puesto que el presente trabajo es de naturaleza sincrónica, no trataré aquí este asunto diaerómico.

\subsection{El factor edad y el empleo pronominal}

Cada personalidad ficticia incluida en el cuestionario se presentó a los informantes acompañada de una edad que iba de 13 a 80 años. Esta amplitud de edades sirve para analizar el efecto de personas entresacadas de tres grupos distintos de edades. Estos tres grupos se clasifican según el siguiente esquema:

$$
\begin{aligned}
& \text { Grupo } I=0-25 \text { años } \\
& \text { Grupo II }=26-50 \text { años } \\
& \text { Grupo III }=51+\text { años }
\end{aligned}
$$

Esta clasificación permite el análisis tanto de personas menores y mayores que los informantes como de las que se encuentran dentro del mismo grupo. Los porcentajes en cuanto a la interacción del factor edad con la nacionalidad y la selección de $T / V$ se presentan a continuación en la tabla 2 (ESP $=$ españoles, $\mathrm{MEX}=$ mexicanos).

En este conjunto de datos notamos que, para cada caso, a medida que aumenta la edad disminuye el empleo de T. Este hallazgo concuerda con otras muchas investigaciones sobre esta especie de sistema pronominal (por ejemplo, con Bates \& Benigni 1975, Lastra de Suárez 1972, Borrego Nieto et al. 1978). 
TABLA 2

$T / V$ según edad de receptor, por nacionalidad.

\begin{tabular}{lcccc}
\hline & $T U ́-E S P$ & $T U^{\prime}-M E X$ & UD. $E S P$ & $U D .-M E X$ \\
\hline Grupo I (0-25) & $80 \%$ & $38 \%$ & $20 \%$ & $62 \%$ \\
Grupo II (26-50) & $49 \%$ & $17 \%$ & $51 \%$ & $83 \%$ \\
Grupo III (51+) & $13 \%$ & $3 \%$ & $87 \%$ & $97 \%$ \\
\hline
\end{tabular}

Sin embargo, la variación que existe entre los españoles y los mexicanos a cada nivel de edad resulta muy llamativa. De hecho, la sugerencia que podría extraerse de estos datos es que los españoles encuestados, como agrupación, consideran la edad un elemento clave para la elección de T/V. Los mexicanos, en cambio, suelen emplear "usted" con individuos pertenecientes a cualquier categoría de edad. Adicionalmente, no sería difícil suponer que existe un continuo de respeto más estratificado dentro de la sociedad española, es decir, el tratamiento a los mayores exige una demostración de deferencia más extrema que el que se ofrece a los menores o a los individuos de semejante edad. Este continuo en el caso mexicano, a diferencia del español, no depende tanto de las diferencias de edad que se obtienen entre los interlocutores; importa más el hecho de conocer o no a la persona.

\subsection{El factor sexo y la elección pronominal}

De las 65 preguntas ofrecidas en la encuesta, unas 32 las constituyeron personalidades femeninas, mientras que 33 fueron representadas por identidades masculinas. Aunque se intentó proveer números iguales de hombres y mujeres en cada clasificación social (véase infra el apartado 4.4), mi meta principal en la elaboración de la encuesta fue facilitar situaciones y personas auténticas y verídicas. Desgraciadamente este propósito no se cumplió, puesto que las posiciones sociales de ambos países todavía no se han abierto a los dos sexos. Por esta razón, me parece legítimo advertir ahora que los resultados conseguidos en este apartado muy posiblemente muestran la influencia de esta carencia de congruencia social. Simplemente, en tanto que los hombres tienden a ocupar empleos más altos que las mujeres en estas dos poblaciones, es probable que ellos reciban un porcentaje más prominente del tratamiento formal. 
Con referencia a los otros trabajos de $\mathrm{T} / \mathrm{V}$ en España que se mencionaron antes, solamente el que investigó el uso de los pronombres en las escuelas de Madrid (Fox 1969) llegó a concluir que el sexo del interlocutor (esto es, la persona a quien habla el informante) influye en la elección de T/V. Sin embargo, las únicas personalidades presentadas a los escolares por Fox eran sujetos que, por lo menos en esa época, exigían el respeto, tales como abuelos y maestros. Por lo tanto, opino que las deducciones a las que llegó Fox gozan de poca validez. El trabajo de Borrego Nieto et al. y el de Aguado Candanedo, que utilizó el mismo cuestionario, descubrieron que las respuestas de universitarios españoles no dependieron del sexo de la otra persona y, así, concluyeron que este factor está mínimamente ligado a la selección de T/V

Por lo que respecta a la investigación previa mexicana, el único trabajo que trata el tema del que nos ocupamos aquí, el breve estudio de Lastra de Suárez (1972), no presenta datos relativos al efecto del factor sexo sobre la elección pronominal. Siendo así, que yo sepa, no existen cifras referentes a este elemento, anteriores al presente trabajo.

Tras analizar las respuestas dadas por los informantes, en la tabla 3 se presentan los resultados obtenidos:

TABl.A 3

$T / V$ seguin sexo de receptor, por nacionalidad.

\begin{tabular}{lcccc}
\hline & $T U ́-E S P$ & $T U ́-M E X$ & $U D .-E S P$ & $U D .-M E X$ \\
\hline mujer & $58 \%$ & $24 \%$ & $42 \%$ & $76 \%$ \\
hombre & $38 \%$ & $12 \%$ & $62 \%$ & $88 \%$ \\
\hline
\end{tabular}

No obstante las advertencias comunicadas arriba de la tabla, al reparar en los datos, aparentemente se señala alguna diferencia de trato pronominal según el sexo del otro individuo incluido en el discurso. Esta observación queda especialmente ratificada en el caso de los españoles, quienes muestran una tendencia para $\mathrm{T}$ con las mujeres, la que difiere del $\mathrm{V}$ que suele emplearse con los hombres. Este hallazgo, además, concuerda con los de otros sociolingüistas como Wolfram (1991) y Fasold (1990) que afirman la propensión muy extensa a pautas más respetuosas cuando la persona a quien se habla es del sexo masculino. En cambio, los mexicanos prefieren $\mathrm{V}$ tanto con hombres como con mujeres. Aunque se aprecia alguna distinción entre los porcentajes prono- 
minales para los dos sexos en el grupo mexicano, yo creo que esta variación, con toda probabilidad, se debe a la incongruencia de papeles que fueron aplicados a las identidades posicionales expuestas en la encuesta (véanse los comentarios supra).

\subsection{El factor clase social $y$ los pronombres $T / V$}

La clase social, como factor interpretativo en la sociolingüística, ha disfrutado de vasta aplicación desde los primeros estudios de Fischer (1958) y Labov (1964). La supervivencia de clase social es también notable a lo largo de la historia de la investigación de los pronombres $\mathrm{T} / \mathrm{V}$, puesto que el trabajo que lanzó esta disciplina al mundo moderno académico, el de Brown y Gilman (1960), contó con este elemento de una manera firme. Ellos no sólo basaron su postulación de las dos "semánticas"' en él, sino que también lo utilizaron para pronosticar cambios futuros en el sistema pronominal bajo estudio.

Los estudios anteriores en cuanto al empleo de T/V en España han llegado, básicamente, a la misma conclusión. Ésta es, en efecto, que la clase social demuestra una influencia bastante grande en la elección pronominal. Sin embargo, tanto el estudio de Borrego Nieto et al. (1978) como el de Aguado Candanedo (1981) aseveran que las consecuencias de clase social en T/V pueden ser anuladas por las de la edad. Es decir, un anciano de 80 años, aunque sea pobre, será tratado con $\mathrm{V}$ en la gran mayoría de las ocasiones. $Y$, si bien los resultados aquí presentados no han sido medidos tomando en cuenta la edad y clase social juntas por limitaciones de espacio, la intuición que se tiene al revisar los cuestionarios es que, efectivamente, ejerce la edad mayor influjo sobre el uso de T/V.

De nuevo, poco se conoce acerca del efecto que practica el componente de clase social sobre los pronombres de segunda persona singular en el territorio mexicano. Lastra de Suárez sí alude a la carencia de influencia dentro de la clase media cuando caracteriza la relación "jefe-subalterno", por medio de un diagrama, como una en la que reina el uso recíproco de $\mathrm{V}$. Desgraciadamente, empero, la autora no señala la clasificación social del superior en la pareja, y, además, no hace mención de la interacción de hablantes de la clase media con miembros de otros niveles socioeconómicos.

Las divisiones de clase social en la presente investigación, al 
igual que las de edad, constan de tres: clase baja (I), clase inedia (II) y clase alta (III) ${ }^{16}$. La separación de identidades posicionales en estas clases se efectuó por dos métodos: primero, siguiendo las clasificaciones hechas por Borrego Nieto et al. (1978), y, segundo, consultando a varios individuos mexicanos y españoles no participantes en este estudio para confirmación actual (y, cuando fue necesario, ajustes) de la ordenación elasificatoria.

Véanse en la tabla 4 los resultados que se alcanzaron.

TABLA 4

$T / V$ según clase social del receptor, por nacionalidad.

\begin{tabular}{lcccc}
\hline & $T U ́-E S P$ & $T U-M E X$ & UD. -ESP & UD. - MEX \\
\hline I (clase baja) & $78 \%$ & $30 \%$ & $22 \%$ & $70 \%$ \\
II (clase media) & $49 \%$ & $21 \%$ & $51 \%$ & $79 \%$ \\
III (clase alta) & $17 \%$ & $5 \%$ & $83 \%$ & $95 \%$ \\
\hline
\end{tabular}

En esta tabla, notamos un fuertísimo efecto de la clase social en la selección pronominal de los españoles. Los porcentajes de ellos, al subir en clase del nivel I al III, por poco se tornan imágenes reversas, en tanto que "tú" con los de clase baja llega a 78\%, mientras que "Ud." con hablantes de clase alta aumenta hasta $83 \%$. Sin embargo, este cambio no se diferencia en gran medida de los resultados considerados en cuanto al factor $e d a d$, mostrados anteriormente en la tabla 2.

Los mexicanos, de manera semejante a la de la tabla 2, no varían grandemente de un nivel a otro. De hecho, su variación a lo largo de la escala de clase es aún menor que la que se encuentra dentro de las edades, sugiriendo que el efecto que ejerce el factor clase social sobre el uso pronominal mexicano es algo aminorado al comparárselo con la influencia de edad.

Ahora bien, con la terminación del estudio de los tres clásicos parámetros sociológicos, sería oportuno un pequeño resumen de algunas tendencias notables de cada grupo nacional hasta este punto.

En términos básicos, los españoles parecen considerar los fac-

${ }^{16}$ Labov (1990) asevera que, al menos, cuatro agrupaciones o divisiones de clase deben hacerse para una división realista y útil de la jerarquía social. En este estudio, no obstante, a causa de la dificultad que se encuentra en el intento de dividir personajes y sus trabajos en cuatro clases, nos hemos limitado únicamente a tres. 
tores sociales de edad, sexo y clase de un modo más notorio que los mexicanos. Para ejemplificar esta inclinación, podemos imaginarnos extremos como la situación de un juez español de 60 años, el cual casi puede asegurarse recibirá trato de V. Mientras, una dependienta española de 16 años probablemente recibirá $T$ de personas semejantes a las que comprenden la presente muestra. Sin embargo, para los españoles, los contextos en que un interlocutor se percibe como igualitario (pero desconocido) presentan un dilema para el hablante, en tanto que el pronombre que se elegirá puede resultar ser $\mathrm{T}$ o $\mathrm{V}$, aunque, aparentemente, hay una preferencia pequeña para este último.

Al reflexionar sobre los resultados mexicanos construidos según la influencia que ejercen los componentes sociales, igualmente patente resalta la carencia de efecto de éstos. A diferencia de los españoles, los mexicanos, al desconocer a una persona, ya sea ésta de cualquiera de las clasificaciones de edad, sexo o clase social, muestran una fuerte disposición a utilizar $\mathrm{V}$ con ese individuo. Efectivamente, el nivel de respuestas $\mathrm{T}$ más alto al cual llega el grupo mexicano es sólo de $38 \%$, cuando el receptor cabe entre las edades de 0 y 25 años. Por esta razón, podemos postular $\mathrm{V}$ como forma prevaleciente entre los mexicanos y $\mathrm{T}$ como forma muy infrecuente, y usual con conocidos o familiares, como veremos a continuación.

\subsection{El uso pronominal dentro de la familia}

Trece preguntas en cuanto al empleo de $\mathrm{T} / \mathrm{V}$ dentro de la familia se hiciéron en nuestro cuestionario. Éstas consistían en un grupo de identidades consideradas como "familia nuclear" (por ejemplo, padres, hermanos) y otro conjunto de personajes categorizado bajo la etiqueta de "familia extendida" (por ejemplo, tíos, primos). No se incluyeron familiares políticos, tales como "suegro" o "nuera". Además, tanto el pronombre "dado"' como el "recibido" fueron reportados por los sujetos. Sin embargo, ya que la gran mayoría de los pronombres "recibidos" eran del tipo T, de hecho $99 \%$ de ellos, no haré aquí el análisis de estos casos. Basta decir que nuestros informantes reciben $\mathrm{T}$ de todos sus familiares.

Los resultados obtenidos, en contraste con otros comentarios afines ya hechos, no varían mucho de los que exhibió Marín (1972) en su estudio comparativo entre hispanoamericanos y es- 
pañoles. También, aunque se hicieron preguntas separadas en cuanto a la madre y al padre, o la abuela y el abuelo, los datos se muestran unificados, así como los de "demás familia nuclear", y "demás familia extendida". Considérense las cifras en la siguiente tabla:

TABLA 5

$T / V$ dado a familiares, por nacionalidad

\begin{tabular}{lcccc}
\hline & $T U ́-E S P$ & $T U ́-M E X$ & $U D .-E S P$ & $U D .-M E X$ \\
\hline padres & $94 \%$ & $84 \%$ & $6 \%$ & $16 \%$ \\
abuelos & $61 \%$ & $78 \%$ & $39 \%$ & $22 \%$ \\
demás familia nu- & & & & \\
clear & $100 \%$ & $100 \%$ & $0 \%$ & $0 \%$ \\
demás familia ex- & $83 \%$ & $95 \%$ & $17 \%$ & $5 \%$ \\
tendida & & & & \\
\hline
\end{tabular}

Los datos anteriores demuestran muy hábilmente que no existe mucha diferenciación entre los dos grupos investigados en su trato pronominal con miembros de la familia. Aunque hay un poco de distinción entre el empleo de $\mathrm{T} / \mathrm{V}$ con los abuelos y la familia extendida, me parece que ésta resulta del hecho de que, como agrupación, los españoles tenían un promedio de edad más alto, cerca de 40 años, mientras que el de los mexicanos era de 34. De nuevo, esta disparidad es debida a la disponibilidad de los informantes y no presenta, a mi ver, ninguna complicación para la exposición del trabajo. En fin, pues, la especulación de que no existen grandes distinciones entre los mexicanos y españoles estudiados, al tratar con la familia, queda confirmada por los resultados aquí presentados.

\section{LA RECIPROCIDAD Y T/V EN CONTEXTO}

Aunque la reciprocidad fue un elemento vital dentro de la teoría original T/V de Brown y Gilman (1960), su inclusión dentro de investigaciones sobre estos pronombres en la lengua española ha sido prácticamente nula. En cuanto a T/V en España o México, por ejemplo, solamente existe un pequeño número de diagramas ofrecidos por Lastra de Suáre $z^{17}$ que perfilan unas cuantas si- 
tuaciones que se dan entre interlocutores de la ciudad de México. Adicionalmente, hay estudios teóricos, como el de Solé (1978), que nos presentan algunos comentarios sobre el tratamiento simétrico y asimétrico en Latinoamérica. No obstante, el campo académico de la sociolingüística carece de aplicaciones empíricas de tales teorías al tema en el habla de informantes verdaderos.

Una investigación sobre pronombres tipo- $\mathrm{T} / \mathrm{V}$ en otros idiomas, como la de Bates y Benigni (1975), ha llegado a la conclusión de que "to summarize the data on reciprocity, we can conclude that differences in age are the only major status differences likely to trigger non-reciprocal address" ${ }^{\prime}$. No obstante esta aserción, en vista de los datos que se han recopilado arriba sobre los efectos de clase social y edad, especialmente en el caso de los españoles, no parece que el comentario de Bates y Benigni conserve su validez en el presente estudio.

Sin embargo, en esta investigación se utilizó otro factor que, aunque nunca se ha empleado anteriormente, aparenta ser pertinente para el estudio del uso de los pronombres T/V. La variable de Contexto Pronominal Anterior (CPA) fue manejada en un intento de extraer otros efectos y distinciones en el uso de estos pronombres. El CPA tiene dos valores: no-existente (-CPA) y existente (+CPA). Sencillamente, el -CPA señala una situación en la cual el informante comienza la plática con otro individuo empleando $\mathrm{T}$ o V. El + CPA, en contraste, simboliza la situación en que otra persona empieza el intercambio con $\mathrm{T}$ o $\mathrm{V}$ y el informante responde con reciprocidad o falta de ella. Esta división de contexto no es, de ninguna manera, insignificante.

Antes de presenciar los efectos del CPA, empero, hace falta ver los datos sobre la reciprocidad generalmente. Treinta y dos preguntas, sin incluir las que trataban los parientes (véanse los comentarios en el apartado 4.5), se hicieron con posibilidad de designar el tema de reciprocidad. Dentro de la sección de -CPA, 12 preguntas en las cuales el informante marcaba tanto el tratamiento "dado" como el "recibido" fueron incluidas. La parte de + CPA, en cambio, se presentó con un total de 20 preguntas, de las que 10 señalaban el $\mathrm{T}$ dado al informante, y $10 \mathrm{el}$ $\mathrm{V}$ dado al informante. Como siempre, la descripción completa de los personajes ficticios, descrita en el apartado 3, acompañaba las preguntas. Los tanteos totales para las situaciones en que la reci- 
procidad podría obtenerse, y efectivamente se obtuvo, aparece en la tabla 6 .

TABLA 6

Reciprocidad según contexto, por nacionalidad

\begin{tabular}{lccc}
\hline & $-C P A$ & + CPA & Todo contexto \\
\hline mexicanos & $85 \%$ & $55 \%$ & $66 \%$ \\
españoles & $79 \%$ & $59 \%$ & $66 \%$ \\
\hline
\end{tabular}

Si consideráramos solamente los datos presentados en la tabla 6 , seguramente llegaríamos a concluir que no se dan diferencias significativas entre los españoles y los mexicanos en cuanto a la reciprocidad del pronombre T/V. Claramente se ve, por ejemplo, que, al considerar todos los contextos, los dos grupos presentan tendencias iguales para reciprocar el pronombre de la otra persona en el discurso. Sin embargo, cuando analizamos reciprocidad por medio tanto del contexto como del pronombre reciprocado, conseguimos resultados muy distintos a los que se ven arriba. Primero, consideremos los datos que se obtuvieron, tomando en cuenta ambos pronombres, cuando no se presentó CPA, esto es, las 12 situaciones en que el informante tuvo la oportunidad de señalar tanto el "dado" como el "recibido"'. Las posibles respuestas son: TR, "tú recíproco”; VR, "usted recíproco"; TNR, "tú no recíproco"; VNR, "usted no recíproco". Los resultados aparecen en la tabla 7:

TABLA 7

$T / V$ dado $\sin C P A$, por nacionalidad

\begin{tabular}{lcccc}
\hline & $7 R$ & $V R$ & $T N R$ & $V N R$ \\
\hline mexicanos & $8 \%$ & $76 \%$ & $3 \%$ & $13 \%$ \\
españoles & $34 \%$ & $44 \%$ & $9 \%$ & $13 \%$ \\
\hline
\end{tabular}

Aquí notamos la manera en que las normas preliminares que habíamos bosquejado al terminar el análisis de los factores sociales (véase el apartado 4.4) ejercen fuerte influencia sobre lo que los informantes consideran el tratamiento ideal entre dos interlocutores. Claro está que en una sección de únicamente 12 preguntas no pueden medirse todos los efectos. Sin embargo, entre estas 
12 preguntas hicimos una división de 6 hombres y 6 mujeres, 4 de cada uno de los tres grupos de edad y 4 de los tres grupos sociales. Así, resultan especialmente interesantes los datos mexicanos, los cuales muestran una tendencia aún más grande que en los datrs globales (apartado 4.1) para el empleo de V. Los españoles, en contraste, aunque sí demuestran alguna variabilidad en este contexto, se acercan mucho más a sus resultados globales que los mexicanos.

En el caso de + CPA, del que constaban 20 preguntas, también se intentó ofrecer casos creíbles e iguales en cuanto a edad, sexo y clase social como en la sección sobre el -CPA. Otra vez, 10 personajes fueron presentados dando $\mathrm{T}$ al informante, al igual que otros 10 dando $\mathrm{V}$. El propósito bajo observación con el empleo de esta variante contextual es que existen factores del escenario que influyen en la elección del pronombre (Irvine 1979, Ervin-Tripp 1964, Labov 1972). Y, como puede imaginarse, consecuencias sobre el monto de casos en que se obtiene la reciprocidad también se derivan de la inclusión del + CPA. Como primer elemento, nótense los resultados obtenidos cuando se daba $\mathrm{T}$ al informante, lo cual representa unas 180 situaciones para los dos grupos estudiados, que aparecen en la tabla 8. Las dos respuestas posibles son "T recíproco" (TR) y "V no recíproco" (VNR).

TABLA 8

$T / V$ dado con $C P A$ de $T$, por nacionalidad

\begin{tabular}{lcc}
\hline & $T R$ & $V N R$ \\
\hline mexicanos & $29 \%$ & $71 \%$ \\
españoles & $64 \%$ & $36 \%$ \\
\hline
\end{tabular}

Estos resultados nos enseñan, de nuevo, la fuerte disposición de los mexicanos a utilizar V, no importa el estatus del otro hablante. Los españoles, mientras, se inclinan a reciprocar el $\mathrm{T}$ y, supongo, emplear $\mathrm{V}$ cuando la edad o la clase social de la persona lo merece. Esta inclinación fue resumida muy bien por un comentario de una española de 23 años no-participante en el estudio, a quien pregunté sobre este hecho: "A nosotros, si nos hablas de tú, no nos importa tu identidad, te responderemos con tú". Por supuesto, los datos no confirman por completo tal aser- 
ción; sin embargo, nos da una idea general sobre la reciprocidad en España, especialmente entre los jóvenes.

Por último, fueron analizados 10 ejemplos de situaciones en que el informante ha recibido trato de V. En este caso, ios sujetos respondieron con "V recíproco" (VR) o "T no recíproco" (TNR). Sus resultados se presentan a continuación, en la tabla 9:

TABLA 9

$T / V$. dado con CPA de $V$, por nacionalidad

\begin{tabular}{lcc}
\hline & $V R$ & $T N R$ \\
\hline mexicanos & $81 \%$ & $19 \%$ \\
españoles & $55 \%$ & $45 \%$ \\
\hline
\end{tabular}

Aquí, como en otras numerosas ocasiones, los mexicanos están muy inclinados a responder con $\mathrm{V}$, a la exclusión virtual de T. Además, aunque los números no se han dividido de tal manera, me parece sumamente probable que los casos de TNR ofrecidos por los mexicanos traten de personas de menor edad, o posición social con las que se dé una relación más informal, por ejemplo, al charlar con el taxista o el conductor de autobús.

Los españoles muestran que la selección de T o V está sujeta a las mismas reglas que aplican cuando se enfrentan a otro hablante sin CPA. Si los elementos de identificación sociológica concuerdan con el empleo de $\mathrm{V}$, es decir, en este caso, la reciprocidad, ellos ofrecerán V como respuesta al V inicial. Si estos componentes clasificatorios no convienen al tratamiento recíproco, el encuestado responderá con $\mathrm{T}$ no recíproco para marcar la diferencia de estatus existente en la situación.

\section{Conclusión}

El presente estudio, efectuado dentro del marco teórico avanzado por primera vez por Brown y Gilman (1960), ha revelado numerosas tendencias en cuanto a la naturaleza del empleo de los pronombres "tú" y "usted" en España y México. Además, ha sido introducida una nueva metodología, la del Contexto Pronominal Anterior, para proporcionar datos más relevantes, confiables y, tam- 
bién, comparables para la investigación del grado de reciprocidad inherente en los sistemas de tratamiento pronominal $\mathrm{T} / \mathrm{V}$.

En cuanto a la caracterización del empleo de los pronombres $\mathrm{T} / \mathrm{V}$ entre los dos grupos, no resulta muy difícil categorizarios respecto a las "semánticas" de "poder" y "solidaridad" sugeridas por Brown y Gilman como prevalecientes en los idiomas que cuentan con este sistema pronominal. Sin duda, los españoles conservan más de la idea del "poder" del interlocutor, en tanto que sus resultados, consistentemente, muestran que los factores sociológicos de edad, sexo y clase social, y su resultante estratificación, afectan la elección de T/V con otro individuo. En cambio, los mexicanos no recurren muy a menudo a tales nociones sociales para su empleo de T/V. En lugar de ellas, este grupo parece apelar sólo al grado de familiaridad (y, pues, "solidaridad") que tiene con una persona dada. Este hecho queda corroborado al observar los datos de $\mathrm{T} / \mathrm{V}$ con parientes, a quienes los mexicanos suelen tratar, a todos, de tú.

Sin embargo, la presente investigación contribuye a más que a la localización de España y México dentro del esquema de Brown y Gilman. Al revisar el vasto conjunto de resultados que se recopilaron, podemos caracterizar los dos países, o, por lo menos, los dos grupos representantes de ellos, en una forma sencilla según el pronombre que más utilizan. Obviamente, como nos lo demostró la primera tabla, T es de uso más frecuente en España, mientras que $\mathrm{V}$ goza de aplicación más extensa en México, en contra de los hallazgos de Lastra de Suárez (1972) resumidos por la cita en la página 131 del presente estudio. No obstante estos datos, también hemos visto que se obtiene más flexibilidad de T/V entre los españoles, y los mexicanos, en contraste, no permiten que la identidad social del otro hablante maneje el cambio entre $\mathrm{T}$ y V. En vista de estas dos tendencias, entonces, es posible concebir un continuo imaginario en que se pueden colocar estos dos países (y, desde luego, otros de habla española). Este continuo puede representarse conforme a la siguiente figura:

Figura 1

Colocación de España y México en el "continuo pronominal"

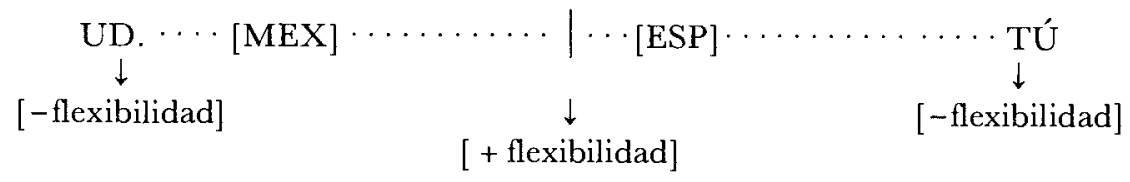


Nos indica este continuo, por medio de las posiciones en que se encuentran los dos grupos, que los informantes mexicanos [MEX], quedan más cercanos al uso de V, o "usted", el cual, en su uso absoluto, no facilita flexibilidad de elección pronominal. Adicionalmente, los españoles [ESP] se localizan próximos al término medio del continuo, donde prevalece más flexibilidad en el uso de T/V según la situación y los interlocutores. El otro punto extremo, que señala el empleo exclusivo de $\mathrm{T}$, o " tú", también permite poca flexibilidad dentro del sistema de T/V. La flexibilidad, además, siempre aumenta a medida que se acerca al punto medio de la escala, y disminuye ai moverse hacia los extremos. Queda bastante claro que los sitios que ocupan España y México no representan sus posiciones fijas, sino que, al agregar otras agrupaciones de hispanoparlantes, podría establecerse un retrato que comparara diversos hablantes con algún ápice de exactitud.

Por último, los significativos resultados que generó la inclusión del factor de Contexto Pronominal Anterior (CPA) evidencian que su aplicación al estudio del tema de los pronombres $\mathrm{T} / \mathrm{V}$ es merecida. Aunque múltiples factores, usualmente de índole sociológica en el plano individual, han sido asignados dentro del campo de los pronombres $\mathrm{T} / \mathrm{V}$, que yo sepa, uno semejante al CPA nunca antes se había ideado. Y, en mi opinión, la introducción de tal elemento al campo no es trivial, en tanto que dentro de la representación de una situación de discurso entre dos personas no puede imaginarse que el mismo individuo inicie la conversación en cada ocasión. Es decir, en resumen, que el CPA sí se da en la vida cotidiana, y nosotros, entonces, como sociolingüistas, nos vemos forzados a incluirlo.

Scott A. Schwenter

University of New Mexico

\section{BIBLIOGRAFÍA}

Aguado Candanedo, D. 1981. "Análisis sociolingüístico del uso de TÚ/USTED, en los estudiantes universitarios de Bilbao", Letras de Deusto, 7, 21, $165-184$.

Alba de Diego, V., y J. Sánchez Lobato 1980. "Tratamiento y juventud en la lengua hablada: aspectos sociolingüísticos", $B R A E, 60,95-129$.

BATES, E., \& L. BENIGNI 1975. "Rules of address in Italy: A sociological survey”, Language in Society, 4, 271-288. 
Beinhauer, W. 1978. El español coloquial. $3^{\text {a }}$ ed. Gredos, Madrid.

Borrego Nieto, J., J. J. Gómez Asencio, y J. A. Pérez Bowie 1978. "Sobre el tú y el usted", Studia Philologica Salmanticensia, 2, 53-69.

Brown, R., \& A. GILMAN 1972. "The pronouns of power and solidarity", en Language and social context. Ed. P. P. Giglioli. Penguin, Harmondsworth, pp. 252-282.

ERVIN-TRIPP, S. 1964. "An analysis of the interaction of language, topic and listener", en Directions in sociolinguistics: The ethnography of communication. Eds. J. J. Gumperz and D. H. Hymes. Holt, Rinehart and Winston, New York, pp. 86-102.

- 1969. "Sociolinguistic rules of address", en Sociolinguitics: Selected readings. Eds. J. B. Pride and J. Holmes. Penguin, Harmondsworth, pp. 255-240.

FAsOLD, R. 1990. The sociolinguistic of language. Basil Blackwell, Oxford.

Fisher, J. L. 1958. "Social influences in the choice of a linguistic variant", Word, 14, 47-56.

Fox, J. 1969. "The pronouns of address in Spanish", en Actes du Xe Congrès International de Linguistes. Academie de la République Socialiste de Roumaine, Bucharest, pp. 685-693.

Gumperz, J. J. 1968. “The speech community”, en Language and social context. Pp. 219-231.

- 1982. Discourse strategies. Cambridge University Press, Cambridge.

IRVINE, J. T. 1979. "Formality and informality in communicative events", en Language in use: Readings in sociolinguistics. Eds. J. Baugh and J. Sherzer. Prentice Hall, Englewood Cliffs, NJ, pp. 211-228.

Keller, G. D. 1974. "Spanish tú and usted: Patterns of interchange", en 1974 Colloquium on Spanish and Portuguese Linguistics. Eds. W. G. Milán et al. Georgetown University Press, Washington, pp. 84-96.

LABOV, W. 1964. "Phonological correlates of social stratification", en Directions in sociolinguistics: The ethnography of communication. Pp. 164-176. 1972. Sociolinguistic patterns. University of Pennsylvania Press, Philadelphia. 1984. "Field methods of the project on linguistic change and variation", en Language in use: Readings in sociolinguistics. Pp. 28-53.

1990. "The interaction of sex and social class in the course of linguistic change", Language Variation and Change, 2, 205-254.

Lastra DE SuÁrez, Y. 1972. "Los pronombres de tratamiento en la Ciudad de México", ALM, 10, 213-217.

MARÍn, D. 1972. "El uso de tú y usted en el español actual", $H, 55,904-908$.

SchweNTER, S. A. 1991. "Diferenciación de la valencia verbal en español: una comparación de los estilos conversacionales de México y España". University of New Mexico [inédito].

Solé, Y. R. 1970. "Correlaciones socio-culturales del uso de «tú/vos" y "usted» en la Argentina, Perú y Puerto Rico", BICC, 25, 161-195.

- 1978. "Sociocultural determinants of symmetrical and asymmetrical address forms in Spanish", H, 61, 940-949.

Wainerman, C. 1976. Sociolingüistica de la forma pronominal. Trillas, México.

Wolfram, W. 1991. Dialects and American English. Center for Applied Linguistics, Washington. 
